\title{
Influence of Goal Contents on Exercise Addiction: Analysing the Mediating Effect of Passion for Exercise
}

\author{
by \\ Álvaro Sicilia ${ }^{1}$ Manuel Alcaraz-Ibáñez ${ }^{1}$, María-Jesús Lirola ${ }^{1}$, Rafael Burgueño ${ }^{1}$
}

\begin{abstract}
Based on the self-determination theory (Deci and Ryan, 1985, 2000), the purpose of this study was to examine the effects of exercise goal contents on exercise addiction, taking into account the mediating effects of passion for exercise. A total of 384 university students (284 men and 100 women; Mage $=20.31, S D=3.10$ ) completed a questionnaire that measured exercise frequency and intensity, exercise goal contents (e.g. intrinsic: social affiliation, health management, skill development; extrinsic: image and social recognition), passion for exercise (e.g. harmonious and obsessive), and exercise addiction. After controlling the exercise frequency and intensity effects, results showed that goal contents did not directly predict exercise addiction. However, mediation analysis showed that goal contents predicted addiction through passion for exercise. These results support a motivational sequence in which extrinsic versus intrinsic goals influence exercise addiction because such goals are positively associated with obsessive passion for exercise and negatively associated with harmonious passion.
\end{abstract}

Key words: exercise, motivation, self-determination theory, dualistic model of passion, university students.

\section{Introduction}

Exercise addiction (EA) involves doing exercise repetitively, frequently and generally stereotypically, which is usually difficult to control or reduce despite being physiologically (e.g. tolerance, abstinence) and/or psychologically (e.g. anxiety, depression) harmful (Symons Downs et al., 2004; Terry et al., 2004). Despite the body of evidence showing the negative consequences associated with this type of addiction, it is still unclear what makes people exercise in such an unhealthy way. Motivation has been described in the literature as a key precursor to exercise addiction (González-Cutre and Sicilia, 2012; Parastatidou et al., 2014) and its analysis could help to understand the EA phenomenon.

The self-determination theory (SDT; Deci and Ryan, 1985, 2000) is a general meta-theory that presents a broad structure for the study of motivation and the human personality, which has been often used to explain the influence of motivational elements in the acquisition of healthrelated behaviours. However, when studying motivation, this theory distinguished between the content (what a person pursues in doing an activity) and the process (why they do it). While the motivational process has received a lot of attention when analysing the motivational factors involved in exercise, the relationship between motivational content and exercise has not been studied so intensively (Standage and Ryan, 2012). This imbalance in the research is hard to understand if one considers that the goal a person has in mind when exercising (e.g. getting healthier, improving physical appearance) is also a key element in understanding the way a person gets involved with that activity. The aim of this study was to analyse the relationship between goal contents in exercise and EA.

Goals Contents in Exercise and EA

Goal contents theory is one of the subtheories that establishes SDT. SDT (Deci and

1 - Universidad de Almería, Facultad de Ciencias de la Educación (Almería), Spain. 
Ryan, 2000) postulates that the goals a person pursues when doing an activity can explain the way that he or she goes about it, so that having different goals when carrying out an activity can condition the well-being and personal development it produces. Indeed, this theory distinguishes between intrinsic and extrinsic goal contents. Intrinsic goals are those where the activity is directed at developing personal potential, values and interests. Extrinsic goals, on the other hand, reflect a desire to achieve benchmarks with external value such as gaining social recognition, giving a good image or having financial success (Kasser and Ryan, 1993, 1996). As such, extrinsic values do not come from oneself but from a type of external pressure accorded to which the behaviour is adopted. Research has showed that the kind of goals pursued could lead to different consequences not only in life in general, but also in particular domains, including exercise (Ingledew and Markland, 2008; Kasser and Ryan, 1996; Sebire et al., 2009, 2011; Vansteenkiste et al., 2004).

Before the goal content theory was developed and integrated into the more general SDT structure, previous research had analysed the effect that the various reasons or motives why people undertook exercise had on the way they engaged with the activity. Indeed, within the exercise context, the terms motives or reasons were used synonymously to those which SDT understands today as the goals that a person can pursue when they get involved in a task (Frederick and Ryan, 1993; Ingledew and Markland, 2008; Ryan et al., 1997). In this vein, previous research supported the idea that exercising following intrinsic or extrinsic goals results in different outcomes. For example, exercising with motives that could be considered extrinsic goals (e.g. to improve appearance) was positively associated with exercise anxiety, physical social anxiety and physical discomfort, and negatively associated with self-esteem (Sebire et al., 2009), while exercise motives that could be considered intrinsic goals (e.g. skill-development or interaction with others) were positively related to self-esteem, psychological wellbeing, satisfying basic psychological needs, attention and fun (Sebire et al., 2009). However, to date there have been no studies considering the possible impact that goal contents could exert on EA, established from the perspective of SDT. Nonetheless, some authors suggest that those people excessively preoccupied with creating an ideal body image usually show a propensity for pathological behaviour such as food disorders and EA (Marzano-Parisoli, 2001). In this regard, some reasons and motives that could be considered as extrinsic goals have been positively associated with EA. For example, Fortier and Farrell (2009) showed that people with higher EA scores reported motives for exercising related to physical appearance and being fit.

Nonetheless, research suggests that the size of the effect concerning the direct relationship between goal content and exercise is low; consequently, goal contents could be considered as the distal predictor of exercise (Ingledew and Markland, 2008; Sebire et al., 2009). Indeed, Sebire et al. (2009) have shown that the way in which exercise is internalised into a person's identity can be a mediator in this relationship. A construct which reflects the way a person internalizes the value attributed to a particular activity and thus submits to it, is that of passion (Parastatidou et al., 2014).

\section{Dualistic Model of Passion}

The SDT suggests that people naturally orientate themselves towards activities that satisfy their needs (e.g. their relationships with other people, autonomy and competence), and that these respond to the more autonomous or selfdetermining motivational processes in how they reflect the presence of internal values and how they align themselves with personal goals (Deci and Ryan, 1985). An internalization process functions optimally when the person identifies the importance of the activity's social value, integrating it into their identity and accepting it as a part of themselves (Deci and Ryan, 2000). Considering the idea that activity's internalisation can be a process more or less controlled by the person, Vallerand (2012) and Vallerand et al. (2003) developed a dualistic model of passion.

The concept of passion could be useful to delve deeper into the process by which addition may be associated with internalised ways of exercising. Indeed, Vallerand (2012) has considered two types of passion up to this point. On the one hand, harmonious passion (HP) has been defined as a process that reflects autonomous internalization of an activity into a 
person's identity. On the other hand, obsessive passion (OP) reflects a controlling form of internalization of an activity within a person's identity. Considering the two types of aforementioned passion, Vallerand (2012) suggests that those people that exhibit a HP control the activity; that is to say, they can decide when and where to do it, acting on their own volition. In this way, the activity is in "harmony" with other aspects of their lives. In contrast, those people that exhibit an OP experience an internal compulsion to involve themselves in the activity, even in situations where the activity is inappropriate. In other words, one could say that an OP causes conflicts with other aspects of life (e.g. work, family).

Until now, the research has associated HP with adaptive consequences, such as well-being and positive emotional experiences, while OP has been associated with less adaptive experiences, such as the appearance of conflict with other facets of life, or the presence of negative emotions (Houlfort et al., 2015; Lafrenière et al., 2013; Lin and Chen, 2016). In line with this, some researchers have suggested that, within the exercise context, EA could have a positive association with OP (González-Cutre and Sicilia, 2012; Paradis et al., 2013). Indeed, it appears logical to think that if the addiction entails a behavioural disorder characterised by the compulsive repetition of an activity, this reflects an obsessive dedication to that activity. Thus, if dedication to exercise reflects an $\mathrm{OP}$, then doing it might start to control other facets of a person's life. Thus, the way in which a person integrates exercise into their identity could help to explain the relationship between goal contents and EA.

\section{Study Objectives}

Motivation has been indicated as a variable that can partly explain EA (Parastatidou et al., 2014). Indeed, motives for exercise such as increasing one's appearance, which could be considered an extrinsic goal, have been positively associated with EA (Fortier and Farrell, 2009). Nonetheless, even though the goal contents might include a key factor that explains EA, this has been seen as a distal antecedent of the exercise and thus should be considered in relation to other motivational factors that can mediate this relationship (Sebire et al., 2009; 2011). Passion for exercise has been shown as a proximal antecedent to EA (Paradis et al., 2013) helping to explain the motivational process whereby the person internalizes this activity. In this way, OP in exercise has been proposed as a variable that can mediate the relationship between motivation and EA (Parastatidou et al., 2014).

The aim of the present study was to analyse the relationship between goal contents and EA, considering the way in which exercise is integrated, via the passion construct, into a person's identity. In particular, after revising the literature, it was hypothesised that: a) intrinsic goal contents would negatively predict EA, while extrinsic goal contents would positively predict $\mathrm{EA} ; \mathrm{b})$ the relationship between contents and EA would be mediated by the type of passion for the exercise; in particular, extrinsic goal contents would positively predict EA through the mediating effects of OP while intrinsic goal contents would negatively predict EA by their negative effect on OP.

A novel feature of the present study is to consider the effect of the differentiated prediction of each intrinsic goal (social affiliation, health management and skill development) and extrinsic goals (image and social recognition) measured with the GCEQ (Sebire et al., 2008). To date, studies using this measure have considered a global value for intrinsic and extrinsic goals, taking the average score into account for each of their sub-factors or considering a relative index that is given by the difference between these average scores (Sebire et al., 2008, 2009, 2011). Although these previous studies have shown that intrinsic rather than extrinsic goals present a greater association with adaptive consequences in the context of exercise, they have not analysed the differential predictive power that each of these exert on exercise-related consequences. The differential analysis of each goal seems interesting because it would support the results of the previous research and, in particular, would indicate that each goal is interpreted by the participants in accordance with the intrinsic/extrinsic goal classification established by the SDT. This question appears to be relevant considering the results of the work by McLachlan and Hagger (2011), which suggested difficulties for the person in identifying particular goals as being intrinsic or extrinsic. McLachlan and Hagger (2011) found that the participants, who 
had previously been asked to select a list of goals that they pursued when exercising in their leisure time, were grouped in significantly differentiated clusters based on the intrinsic or extrinsic goals. However, when a subsample of these participants were given a definition of intrinsic or extrinsic motivation, and were then required to mark the goals on the list that they considered to be of one or other type, the results showed that the participants found this difficult to do.

\section{Methods}

\section{Participants}

The study included a sample of 384 university students (284 men and 100 women) who studied the Physical Activity and Sport Science degree. They were selected by nonprobabilistic convenient sampling. These subjects had all exercised regularly over the previous six months. The participants' age was between 17 and $42\left(M_{\text {age }}=20.31, S D=3.10\right) .21 .9 \%$ of the subjects included in the study carried out exercising 2-3 times per week, $56.2 \%$ of them did it $4-5$ times per week and the remaining $21.9 \%$ exercised 6-7 times a week. $51.6 \%$ of the participants usually carried out exercise at moderate intensity and $48.4 \%$ at strenuous intensity, while none of the participants indicated that they carried out this activity at light intensity. Following the criteria established by Terry et al. (2004), 9.1\% of the subjects included in the study were considered asymptomatic, $76.8 \%$ as symptomatic and the remaining $14.1 \%$ as atrisk of EA.

\section{Measures}

Frequency and intensity of exercise.

To collect information on the frequency of physical practice, students reported the number of days a week on which they normally exercised in their free time for at least 15 minutes. At the same time, the participants indicated, according to their subjective evaluation, if the intensity with which they carried out exercise was light, moderate or strenuous in accordance with the proposal of Godin and Shephard (1985).

Goal contents in exercise. The Spanish version (Sicilia et al., in press) of the Goal Content for Exercise Questionnaire (GCEQ; Sebire et al., 2008) was used. This tool comprises 19 items grouped in five sub-scales, three of which collect goal contents which are intrinsic in character, such as social affiliation (4 items; e.g. "to form close bonds with others"), health management (4 items; e.g. "to improve my overall health") and skill development (4 items; e.g. "to acquire new exercise skills"). The two remaining sub-scales collect goal contents that are extrinsic in character, in particular those referring to image ( 3 items; e.g. "to improve my appearance") and social recognition (4 items, e.g. "to gain favourable approval from others"). The scale was preceded by the sentence "please indicate to what extent these goals are important for you while exercising" A Likert scale ranging from 1 (not important) to 7 (very important) was used. The factors included in the instrument had previously shown adequate internal consistency $(\alpha \geq .85)$ and temporal stability (intra-class coefficient, ICC $\geq$ .70) over a two-week period (Sicilia et al., in press).

Passion for exercise.

The Spanish version (Alcaraz-Ibáñez et al., in press) of the Passion Scale in Exercise (PSE; Parastatidou et al., 2012) was used. The PSE comprises 14 items, grouped in 2 sub-scales of 7 items each that evaluate the degree of harmonious (e.g. "Exercise in harmony with other activities in my life") and obsessive (e.g. "I cannot live without exercise") passion of people exercising with regard to the said activity. A Likert scale ranging from 1 (totally disagree) to 7 (totally agree) was used.

Exercise addiction.

The Spanish version (Sicilia et al., 2013) of the Addiction Inventory (EAI; Terry et al., 2004) was used. The scale comprises six items grouped in a single factor (e.g. "Conflicts have arisen between me and my family and/or my partner about the amount of exercise I do"). The sentence that preceded the scale was: "How much do you agree with the following statement?" A Likert scale ranging from 1 (totally disagree) to 5 (totally agree) was used.

\section{Procedures}

Two Spanish universities collaborated in the study. The questionnaire was administered in the presence of one of the project's participant researchers who reported on the characteristics of the study and the voluntary character of the participation. The students did not receive any compensation as a result of their participation . The researcher was available to the participants to resolve any doubts that might appear during 
the data collection process. The approximate time to complete the questionnaire was $20 \mathrm{~min}$. This research was approved by the ethics committee of the researchers' university.

\section{Statistical Analysis}

First, the descriptive statistics and the bivariate correlations between the distinct variables were analysed, as well as the internal consistency of each scale. Second, a stepwise linear regression analysis was carried out to determine the variables that would predict EA. Given that the literature had related EA with the frequency and intensity of practice (Symons Downs et al., 2004), both variables were introduced in Step 1 with the intention of controlling their possible effects. The distinct goal contents were introduced in Step 2, while the harmonious and obsessive forms of passion were introduced in the last step. Third, multiple parallel mediation analyses were carried out using the Model 4 of 'PROCESS' macro for the statistical package IBM SPSS 22, in accordance with the procedure described by Hayes (2013). The bootstrapping procedure used generated 10000 samplings that made it possible to obtain 95\% bias-corrected confidence intervals $(\mathrm{BcCI})$, which indicate the existence of a significant indirect effect in the case of not containing the zero. The effects of all the variables involved in the analysis on EA (frequency and intensity of exercise included) were controlled for. A significance level of $p<.05$ was used for all the statistics.

\section{Results}

\section{Descriptive Statistics}

Table 1 presents means, standard deviations and bivariate correlations for the considered variables, as well as the internal consistency coefficients for the scales used. The mean score for the distinct variables was in all cases above the mid-point of the respective scales. The intrinsic goal content scores (social affiliation, skill development and health management) were above those corresponding to the extrinsic goal contents (image and social recognition). Furthermore, the mean score for HP was above the mean score for OP. The internal consistency indices were above the .70 cut-off point suggested by Nunnally and Bernstein (1994) with the single exception of EAI, which showed a value of .67. In spite of this, it should be noted that the latter value is in line with those obtained by the EAI in previous studies, in similar populations as those considered here (Griffiths et al., 2015). All the considered variables correlated positively with EA, showing intensities that ranged from weak to moderate. These correlations were statistically significant in all cases, with the single exception of HP.

\section{Linear Regression}

In Step 1, both frequency $(\beta=.19, p<.001)$ and intensity $(\beta=.15, p=.005)$ of exercise significantly predicted EA, accounting for $7 \%$ of its variance. In Step 2 , frequency $(\beta=.18, p<.001)$ and intensity $(\beta=.19, p=.005)$ of practice, as well as goal contents regarding image $(\beta=.14, p=.017)$ and social recognition $(\beta=.15, p=.008)$ significantly predicted EA. Predictor variables introduced in Step 2 accounted for 13\% of the EA variance. In the last step, only frequency of practice $(\beta=.10, p=.04)$, harmonious passion $(\beta=$ $-.19, p<.001)$, and obsessive passion $(\beta=.49, p<$ $.001)$ significantly predicted EA. Predictor variables introduced in Step 3 accounted for 32\% of the EA variance.

\section{Mediation Analysis}

Figure 1 shows how once the effects of the intensity and frequency of weekly practice are controlled, the goal contents relating to social affiliation (path $a=.11, p=.003$ ), skill development (path $a=.26, p<.001$ ) and image (path $a=-.10, p=.001$ ) predicted HP in a statistically significant way. For its part, the goal contents regarding health management (path $a=$ $.24, p=.032$ ), skill development (path $a=.38, p<$ .001 ) and social recognition (path $a=.12, p=.003$ ) predicted OP in a statistically significant way. In turn, HP negatively predicted EA (path $b=-.21, p$ $<.001$ ) while positively predicting OP (path $b=$ $.28, p<.001$ ). The remaining effects did not achieve the established level of statistical significance $(p<.05)$.

Table 2 shows the estimation, the standardised error and the $\mathrm{BCCI}$ of $95 \%$ of the goal contents indirect effects on EA through HP and OP. These effects were produced in a statistically significant way through both HP (social affiliation, path $a b=$ $.02,95 \%$ BcCI: -.0484 to -.0079 ; skill development, path $a b=-.06,95 \%$ BcCI: -.1046 to -.0236 ; image, path $a b=.02$, BcCI: .0070 to .0412) and OP (health management, path $a b=-.07,95 \%$ BcCI: -.1359 to - 
.0048; skill development, path $a b=11,95 \% \mathrm{BcCI}$ : .0566 to .1721 ; social recognition, path $a b=.03$, BcCI: .0099 to .0620). The partial effects of the controlled variables on EA were: path $c^{\prime}=.08(p=$ $.22)$ for intensity and path $c^{\prime}=.10(p=.04)$ for frequency of practice. The multiple mediation analysis was shown to be statistically significant $(F[9,374]=19.92, p<.001)$, explaining $32 \%$ of the EA variance.

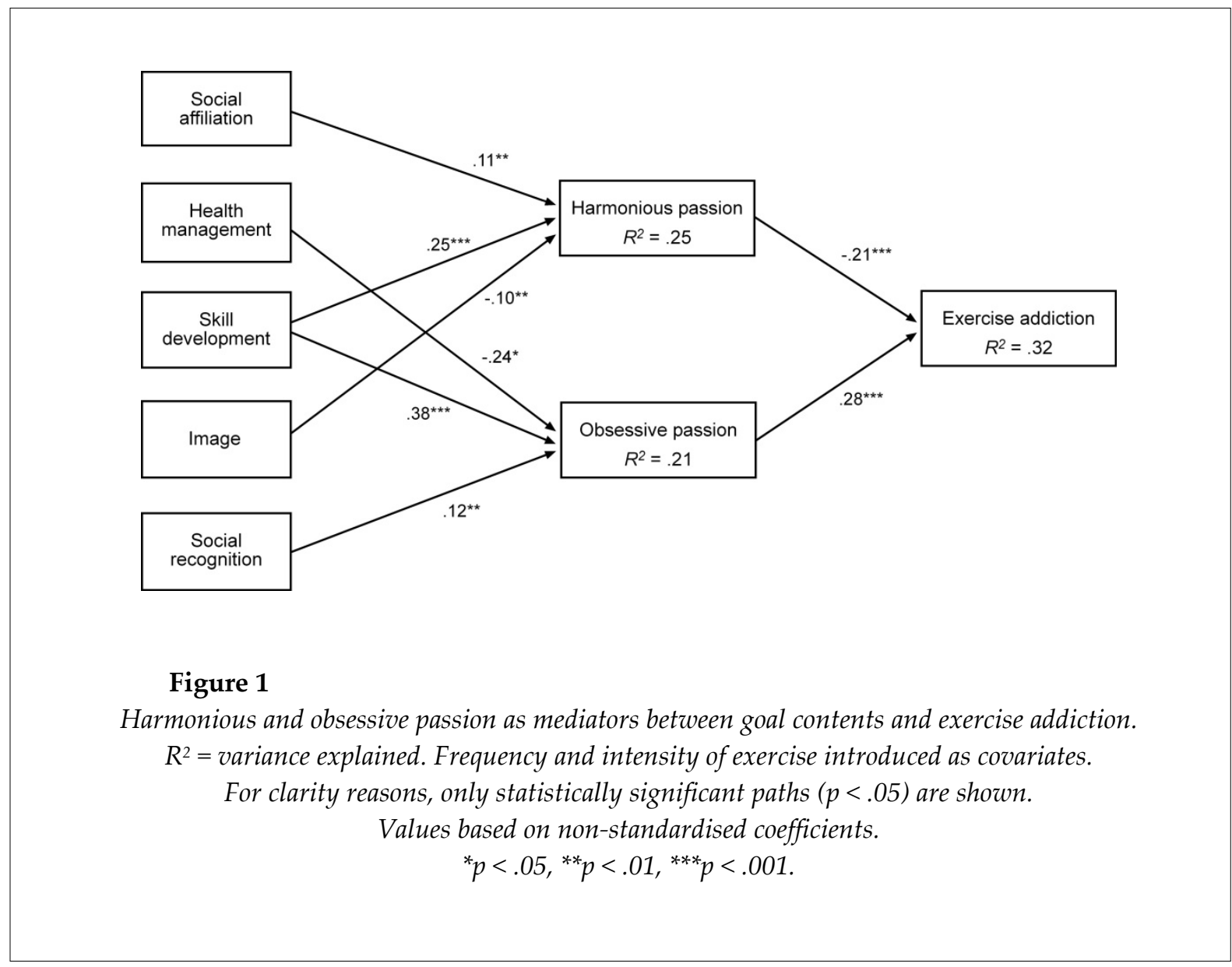

\begin{tabular}{|c|c|c|c|c|c|c|c|c|c|c|c|}
\hline \multicolumn{12}{|c|}{$\begin{array}{c}\text { Table } \mathbf{1} \\
\text { Means, Standard Deviation, Internal Consistency }(\alpha) \text { and Bivariate Correlations of } \\
\text { Main Study Variables }\end{array}$} \\
\hline & $M$ & $S D$ & $\alpha$ & 1 & 2 & 3 & 4 & 5 & 6 & 7 & 8 \\
\hline 1. Social affiliation & 5.98 & .93 & .87 & - & & & & & & & \\
\hline 2. Health management & 6.30 & .75 & .80 & $.54^{* * *}$ & - & & & & & & \\
\hline 3. Skill development & 6.25 & .78 & .84 & $.55^{* * *}$ & $.72^{* * *}$ & - & & & & & \\
\hline 4. Image & 5.34 & 1.11 & .77 & $.33^{* * *+}$ & $.41^{* * *}$ & $.32^{* * *}$ & - & & & & \\
\hline 5. Social recognition & 3.91 & 1.51 & .88 & $.26^{* * *}$ & .08 & $.16^{* * *}$ & $.41^{* * *}$ & - & & & \\
\hline 6. Harmonious Passion & 6.22 & .58 & .75 & $.32^{* * *}$ & $.29^{* * *}$ & $.40^{* * *}$ & .00 & .08 & - & & \\
\hline 7. Obsessive Passion & 4.83 & 1.16 & .86 & $.12^{*}$ & $.10^{*}$ & $.22^{* * *}$ & $.12^{* *}$ & $.22^{* * *}$ & $.35^{* *}$ & - & \\
\hline 8. Exercise addiction & 3.15 & .67 & .67 & $.10^{*}$ & $.13^{*}$ & $.16^{* *}$ & $.20^{* * * *}$ & $.22^{* * *}$ & .06 & $.52^{* * *}$ & - \\
\hline \multicolumn{12}{|c|}{${ }^{*} p<.05 ;{ }^{* *} p<.01 ;{ }^{* * *} p<.001$} \\
\hline
\end{tabular}




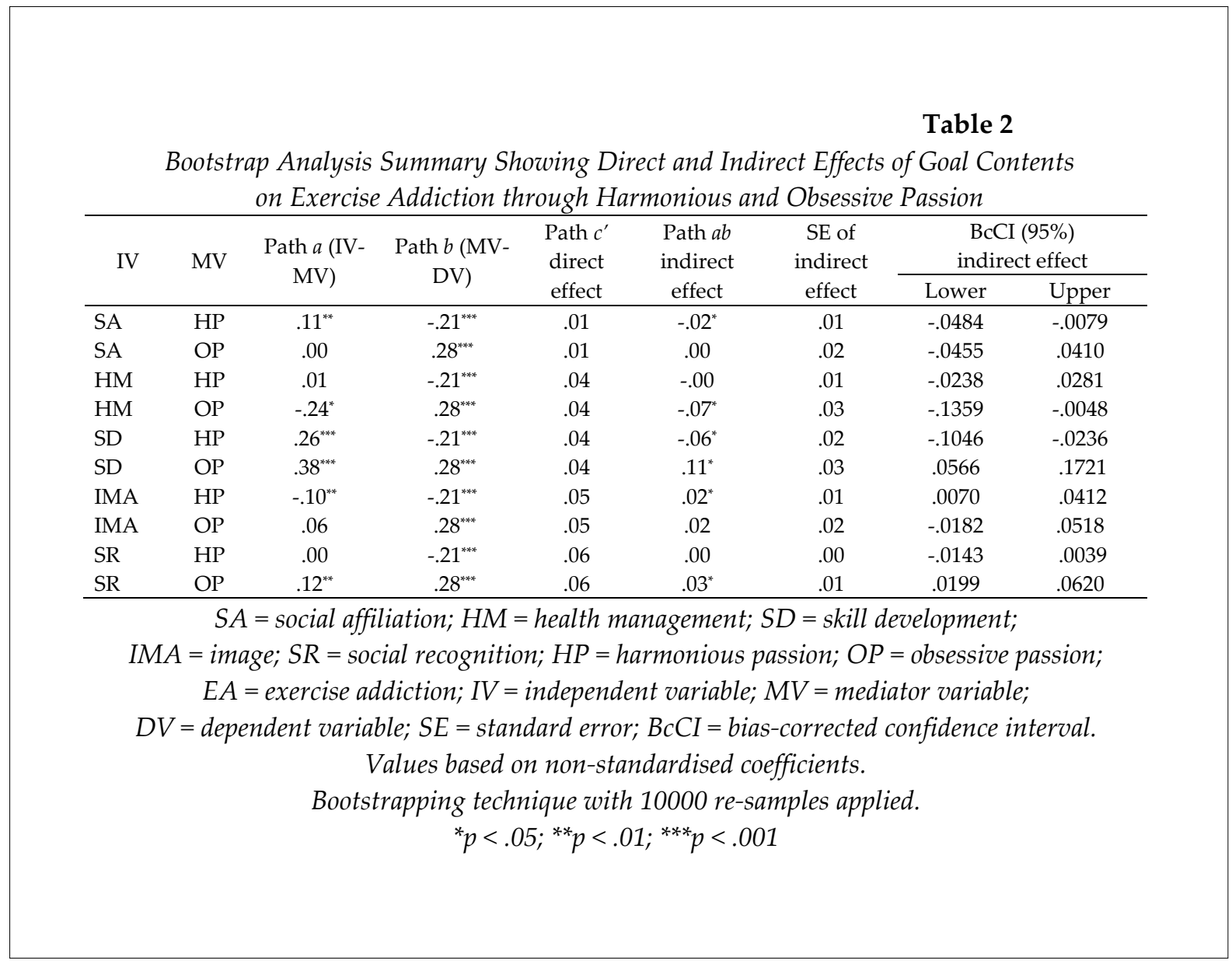

\section{Discussion}

The aim of this study was to analyse if, considered individually, the goals that a person pursues when performing exercise predict addiction to this activity. Furthermore, the possibility was considered that the relationship between goal contents and EA might be mediated by the way the person integrates these activities into their identity (i.e. obsessive or harmonious passion). The results showed that the goal contents predicted EA through the distinct forms of passion for the exercise, namely, negatively in the event of intrinsic contents and positively in the event of extrinsic contents. Nonetheless, the intrinsic goal content referring to skill development predicts EA both negatively through $\mathrm{HP}$ and positively through OP.

In line with previous work (Fortier and Farrell, 2009; Paradis et al., 2013; Parastatidou et al., 2014), the bivariate correlation analysis (Table 1) showed that EA presented moderately or highly positive correlations to the two extrinsic goal contents (e.g. image and social recognition) and OP. Moreover, the linear regression analysis showed that the two extrinsic goals had direct predictive and positive effects on EA when the effect of forms of passion was not considered. However, both the results of the third regression analysis step and of the mediation analysis showed a reduction in the magnitude of the direct effects of the goal contents on EA, as well as a rise in the variance explained for this last variable (13 vs $32 \%$ ).

These results suggest that the effects of goal contents on passion were mediated by the type of passion for exercise. Nonetheless, the way in which each passion type mediated the relationship was clearly different. In particular, while HP showed a statistically significant negative relationship to EA, OP predicted EA in a statistically significant positive way. The results from this study support evidence from previous research regarding the mediating role of passion 
and suggest motivational sequence in which the goal contents influence EA depending on the character of the process through which the person internalises the exercise's attributed value into their identity. Nonetheless, according to our results, both types of passion appear to play a mediating role in the relationship between the goal contents and EA. In particular, the intrinsic goal contents would indirectly and negatively predict EA either because they facilitate the autonomous internalisation process and a harmonious commitment to the exercise (e.g. exercising to make friends and interact) or because they lessen the controlled character of the internalisation process and the obsessive commitment to this activity (e.g. exercising to improve one's health). In contrast, this study's results suggest that when a person pursues extrinsic exercise goals, they would indirectly favour addiction to this behaviour, either because it facilitates obsessive commitment to the exercise (e.g. exercising to gain recognition amongst others) or because it might reduce the integration of the exercise into their identity in a harmonious way (e.g. exercising to improve one's appearance).

The mediation analysis results were partially in line with our hypothesis and the results of previous studies (Houlfort et al., 2015; Lafrenière et al., 2013; Paradis et al., 2013; Parastatidou et al., 2014; Wang and $\mathrm{Chu}, 2007)$, which identified OP as a precursor to addiction in various contexts, including exercise. Nonetheless, our results also showed a mediating effect of HP although of a lesser intensity compared to that of OP, when the relationship between goal contents and EA was analysed. These results were unexpected given the weak or null predictor effect that the previous studies had given to HP on behavioural addictions. Future studies should look more deeply into the possible role that HP can have when predicting EA from different motivational factors.

In addition, the results showed that a goal that was considered intrinsic (e.g. skill development) had both indirect positive and negative effects on EA, what might initially seem contradictory. Specifically, the skill development goal predicted EA in an indirect and negative way through a positive association with OP. This result is surprising as it seems to contradict previous research (Sebire et al., 2008, 2009, 2011;
Vansteenkiste et al., 2004) and the hypothesis posed in this study, in that the intrinsic goals tend to relate positively with adaptive consequences for a person's development. One possible explanation might be that participants understood the skill development goal in different ways. Indeed, previous research, using the same tool to measure goal contents (Sebire et al., 2008, 2009, 2011), did not analyse the individual effects of each goal, so they were limited in their observations if the differential consequences of the intrinsic and extrinsic goal contents complied with each of the sub-factors considered. Furthermore, this possible explanation would be in line with the study results found by McLachlan and Hagger (2011), which suggested that people usually differentiated more easily between intrinsic and extrinsic goals when they did it globally and implicitly than when they did it in a precise and explicit way.

Taking into account the results of the study by McLachlan and Hagger (2011), it is probable that some participants may have interpreted the skill development goal as a controlling factor instead of one that pursues a person's development and self-fulfilment. In fact, some of the items used in the tool that measures this construct indicate the goal of improving their own skill (e.g. to develop my exercise skills; to become skilled at a certain exercise or activity). However, it does not show the person's orientation in determining the achievement of this goal. In this regard, the achievement goal theory (Duda and Nicholls, 1992) considers that in the achievement environment, like the context for exercise, a person can orientate success evaluation in two different ways. On the one hand, the person can value their skill development as a function of a self-referential criterion or, on the other hand, considering their improvement by comparing with the rest. This double orientation when evaluating the skill development goal could explain the contradictory results found in this study. Nonetheless, although this explanation is conceptually plausible, future studies ought to determine if the possible orientation that the person establishes when valuing the skill development goal would determine the appearance of differential consequences. If this were the case, the intrinsic skill development goal could actually represent an extrinsic orientation 
when this goal's evaluation is carried out with normative criteria by others.

In any case, it is important to highlight the key role that the exercise internalisation process plays in the person's identity. Indeed, a reduction in the direct effect magnitude of the goal contents observed, after considering the role of the types of passion, could suggest that the goals that a person pursues when exercising do not play an important part in predicting EA. However, the fact that goal contents predict EA through the types of passion they generate, does not reduce the goals to a valueless variable. Instead, the specific goals that a person pursues when exercising seem valuable owing to the impact they can have on how the person commits to the exercise (e.g. in an obsessive or harmonious way). This would align with the idea that goal contents in exercise appear to play a more distal role, orientated to a more distant future in respect to the way the person gets involved in the exercise. Moreover, in agreement with research showing evidence of different domains in a person's life, this study supports the postulates of the goal content theory and its integration into the SDT (Deci and Ryan, 2000). Furthermore, introducing the concept of passion for exercise within a motivational process that would trigger the risk of exercising in an addictive way, it allows us to see the quality of the goal, and not only its quantity, in the sense that the promotion or pursuit of goals could involve a different way of relating to the exercise, some of which result in a greater addiction risk.

Despite the evidence found in this study, some limitations ought to be highlighted. Firstly, we should bear in mind that the participants in this study were university students of sport science and they were recruited employing a nonprobabilistic method. Thus, the results of this study should be interpreted with caution. Furthermore, the associations analysed in this study should be examined in other student sample groups and more diverse populations. Secondly, this was a transversal study; hence it represents only a snapshot of the association between variables. Longitudinal studies on exercise ought to take place to capture the dynamic nature of the motivational process and EA. As previous studies have established, EA is not something that occurs from one day to another; instead, it is prolonged and the motivational process plays an important role in it (Sussman and Sussman, 2011). Finally, this study considered EA globally; however, this condition presents various symptoms that do not always develop and manifest themselves in the same way in all people. Thus, they can surely acquire different subtleties and phases depending on the physical activity being performed. In fact, an addiction to running or a long-distance trial (e.g. marathon, triathlon, ironman), where there might be a search more for specific effects (e.g. mood changes) is probably different than the addiction to bodybuilding, where a preoccupation for one's appearance might operate more. Future research could analyse the goal contents and the motivational processes that determine addiction to different physical/sport activities.

\section{Acknowledgements}

This research was supported by the Spanish Ministry of Education, Culture, and Sport (FPU-MED).

\section{References}

Alcaraz-Ibáñez M, Sicilia A, Lirola MJ, Burgueño R. Effects of satisfaction and thwarting of basic psychological needs on the forms of passion for exercise. Psychol, Soc \& Educ, 2016; 8: 257-272

Deci EL, Ryan RM. Intrinsic motivation and self-determination in human behavior. New York: Springer Science and Business Media; 1985

Deci EL, Ryan RM. The "what" and "why" of goal pursuits: Human needs and the self-determination of behavior. Psychol Inquiry, 2000; 11: 227-268

Duda JL, Nicholls JG. Dimensions of achievement motivation in schoolwork and sport. J Educ Psychol, 1992; 84: 290-299 
Fortier MS, Farrell RJ. Comparing self-determination and body image between excessive and healthy exercisers. Hellenic J Psychol, 2009; 6: 223-243

Frederick CM, Ryan RM. Differences in motivation for sport and exercise and their relations with participation and mental health. J Sport Behav, 1993; 16: 124-146

Godin G, Shephard RJ. A simple method to assess exercise behavior in the community. Canadian J Appl Sport Sci, 1985; 10: 141-146

González-Cutre D, Sicilia A. Motivation and exercise dependence: A study based on self-determination theory. Res Quart Exercise Sport, 2012; 83: 318-329

Griffiths MD, Urbán R, Demetrovics Z, Lichtenstein MB, de la Vega R, Kun B, Ruiz-Barquín R, Youngman J, Szabo A. A cross-cultural re-evaluation of the Exercise Addiction Inventory (EAI) in five countries. Sport Med - Open, 2015; 1: 1-7

Hayes AF. Introduction to mediation, moderation, and conditional process analysis: A regression-based approach. New York, NY: Guilford Press; 2013

Houlfort N, Fernet C, Vallerand RJ, Laframboise A, Guay F, Koestner R. The role of passion for work and need satisfaction in psychological adjustment to retirement. J Vocat Behav, 2015; 88: 84-94

Ingledew DK, Markland D. The role of motives in exercise participation. Psychol Health, 2008; 23: 807-828

Kasser T, Ryan RM. A dark side of the American dream: Correlates of financial success as a central life aspiration. J Pers Soc Psychol, 1993; 65: 410-422

Kasser T, Ryan RM. Further examining the American dream: Differential correlates of intrinsic and extrinsic goals. Pers Soc Psychol Bulletin, 1996; 22: 280-287

Lafrenière MAK, Vallerand RJ, Sedikides $C$. On the relation between self-enhancement and life satisfaction: The moderating role of passion. Self Identifity, 2013; 12: 597-609

Lin CP, Chen YF. Modeling team performance: The moderating role of passion. J Leadership Organ Stud, 2016; 23: $96-107$

Marzano-Parisoli MM. The contemporary construction of a perfect body image: Bodybuilding, exercise addiction, and eating disorders. Quest, 2001; 53: 216-230

McLachlan S, Hagger MS. Do people differentiate between intrinsic and extrinsic goals for physical activity? J Sport Exercise Psy, 2011; 33: 273-288

Nunnally JC, Bernstein IH. Psychometric theory. New York: McGraw-Hill; 1994

Paradis KF, Cooke LM, Martin LJ, Hall CR. Too much of a good thing? Examining the relationship between passion for exercise and exercise dependence. Psychol Sport Exercise, 2013; 14: 493-500

Parastatidou IS, Doganis G, Theodorakis Y, Vlachopoulos SP. Exercising with passion: Initial validation of the Passion Scale in exercise. Measurement Physical Educ Exercise Sci, 2012; 16: 119-134

Parastatidou IS, Theodorakis Y, Doganis G, Vlachopoulos SP.The Mediating role of passion in the relationship of exercise motivational regulations with exercise dependence symptoms. Int J Mental Health Addiction, 2014; 12: 406-419

Ryan RM, Frederick CM, Lepes D, Rubio N, Sheldon KM. Intrinsic motivation and exercise adherence. International J Sport Psychol, 1997; 28: 335-354

Sebire SJ, Standage M, Vansteenkiste M. Development and validation of the goal content for exercise questionnaire. J Sport Exerc Psychol, 2008; 30: 353-377

Sebire SJ, Standage M, Vansteenkiste M. examining intrinsic versus extrinsic exercise goals: Cognitive, affective, and behavioral outcomes. J Sport Exerc Psychol, 2009; 31: 189-210

Sebire SJ, Standage M. Vansteenkiste M. Predicting objectively assessed physical activity from the content and regulation of exercise goals: Evidence for a mediational model. J Sport Exerc Psychol, 2011; 33: 175197 
Sicilia A, Alcaraz-Ibáñez M, Lirola MJ, Burgueño R. Psychometric properties of the Spanish version of the Goal Content for Exercise Questionnaire. Rev Lat Am Psicol, 2017; pre-print

Sicilia A, Alías-García A, Ferriz R, Moreno-Murcia JA. Spanish adaptation and validation of the Exercise Addiction Inventory (EAI). Psicothema, 2013; 25: 377-383

Standage M, Ryan RM. Self-determination theory and exercise motivation: Facilitating a self-regulatory process to support and maintain health and well-being. In GC Roberts, DC Treasure (Eds), Advances in motivation in sport and exercise. Champaign, IL: Human Kinetics, 233-270; 2012

Sussman S, Sussman AN. Considering the definition of addiction. Environ Res Public Health, 2011; 8: 40254038

Symons Downs D, Hausenblas HA, Nigg CR. Factorial validity and psychometric examination of the exercise dependence scale-revised. Meas Phys Educ Exercise Sci, 2004; 8: 183-201

Terry A, Szabo A, Griffiths M. The exercise addiction inventory: a new brief screening tool. Addict Res Theory, 2004; 12: 489-499

Vallerand RJ. The role of passion in sustainable psychological well-being. Psychological well-Being. Theory Res Pract, 2012; 2: 1-21

Vallerand RJ, Blanchard C, Mageau G, Koestner R, Ratelle C, Leonard M, Gagné, M, Marsolais J. Les passions de l'ame: on obsessive and harmonious passion. J Pers Soc Psychol, 2003; 85: 756-67

Vansteenkiste M, Simons J, Soenens B, Lens W. How to become a persevering exerciser? Providing a clear, future intrinsic goal in an autonomy-supportive way. J Sport Exercise Psy, 2004; 26: 232-249

Wang CC, Chu YS. Harmonious passion and obsessive passion in playing online games. Soc Behav and Personal, 2007; 35: 997-1006

\title{
Corresponding author:
}

\author{
Álvaro Sicilia Camacho, \\ Universidad de Almería, \\ Facultad de Ciencias de la Educación, \\ Carretera de Sacramento s/n, 04120 La Cañada de San Urbano (Almería), Spain. \\ Tel: +34+950 0155394, \\ Fax: +34+950 015751 \\ E-mail: asicilia@ual.es
}

